\title{
High-fidelity simulation of drop collision and vapor-liquid equilibrium of van der Waals fluids
}

\begin{abstract}
The availability of a method to accurately predict the interaction of fuel drops and vapor-liquid equilibrium is crucial to the development of a predictive spray combustion model. The objective of this paper is to present such a method. A numerical method, based on the smoothed particle hydrodynamics (SPH), was coupled with a cubic equation of state for simulating the fuel drop dynamics and liquidvapor distributions at various temperatures in the present study. SPH is a Lagrangian particle-based method, which is useful to simulate the dynamics of fluids with large deformations without the need for a transport equation to track the interface. The present study, furthermore, coupled SPH with van der Waals equation of state to simulate the phenomena of liquid oscillation, drop collisions at high velocity and characteristics of vapor-liquid equilibrium. This approach was found to offer the convenience of using a single set of equations, without the need for submodels, to predict drop breakup or vaporization. A hyperbolic spline kernel function was employed to eliminate the tensile instability that often has been reported in the literature. The numerical method presented here was found to successfully model the merging, stretching separation, fragmentation, and generation of secondary droplets in high-velocity collisions. In predicting vapor-liquid equilibrium, a variable-smoothing-length function was implemented to better facilitate the evaluation of vapor density at low temperatures. Finally, the results of this study indicate that, as the critical temperature was approached, no clear distinction was observed between the liquid and gas phases.
\end{abstract}

Keywords: Drop collision; van der Waals fluid; smoothed particle hydrodynamics; vapor-liquid equilibrium 


\section{Introduction}

The hydrodynamics and vaporization of liquid fuel drops play an important role in the combustion process. The interactions of high-velocity fuel drops affect the distribution of combustible mixtures and, consequently, the combustor performance. These phenomena are often simulated using criteria that predict the outcomes, based on the use of non-dimensional parameters. High-fidelity simulations of fuel drop dynamics can provide useful insight into the fundamental physics of such processes.

In this context, the smoothed particle hydrodynamics (SPH) method has the potential to simulate such phenomena in detail. SPH is a Lagrangian particle-based method, which uses kernel interpolation to evaluate fluid properties at any location. SPH was initially developed for astrophysics simulation [1, 2]. Additionally, SPH has been successfully utilized to solve other fluid dynamics problems, such as shock, ocean wave formation and breakup, and solid mechanics phenomena, such as fracture and fragmentation $[3,4]$. Use of the SPH formulation in solving these types of fluid dynamics problems is particularly effective because it does not require an explicit definition of the free surface location, and thereby allows large deformations to be simulated in greater detail.

Furthermore, the SPH formulation can incorporate the equation of state to describe the vaporliquid equilibrium [5]. This is useful, particularly, because it does not necessitate separate sets of equations to model the liquid and vapor phases. The cohesive term of the pressure equation manifests itself in the form of surface tension at the liquid-vapor interface, and generates phase boundaries without the explicit description of their location or curvature [5]. It was observed that particle rings were formed in the liquid phase at low temperature conditions, a phenomenon attributed to the SPH tensile instability [6]. Tensile instability has been shown to occur when the product of the second-order derivative of the

kernel function and the principal stress is positive [6]. This instability causes particles to agglomerate or disperse away during simulation. A previous study has demonstrated that such instability can be reduced by adding an artificial pressure term in the momentum equation when the local pressure was negative 
[3]. This same methodology was applied to simulate the bending of solid beams [4] and the motion of van der Waals (vdW) fluids [7, 8]. It was also suggested that a kernel function with only positive second-order derivatives (e.g., hyperbolic spline) can ensure that the simulation is stable in compression $[6,9]$.

In predicting vapor-liquid equilibrium, deviations in saturated vapor densities at low temperatures can occur when the kernel smoothing length is a fixed value [5]. In a sparse medium, particles tend to have very little, or no, interaction with neighboring particles, because the latter are located beyond the compact support of the smoothing kernel. Previous studies predicted the spinodal decomposition of non-equilibrium square drops into two stable phases by use of a Korteweg stress tensor, a form of a body force generated by density gradients [10, 11]. A variable smoothing length for better kernel evaluation was used in the vapor phase to obtain equilibrium conditions, closely matching those of the binodal curve. In a study, binary collisions of two infinitely long cylindrical vdW liquids were simulated using SPH, but the collisions were limited to small impact energy with $23<\operatorname{Re}<68$ and $1<\mathrm{We}<10$ [12]. It is apparent that new formulations, based on SPH, are required to accurately predict the outcomes of drop collisions and vapor-liquid equilibrium for combustion application.

In this paper, a numerical method based on $\mathrm{SPH}$, coupled with cubic equations of state, was developed and applied to simulate the dynamics of a vdW drop at high Reynolds number (Re) and Weber number (We). One of the novelties of this work was the implementation of a hyperbolic kernel function combined with a variable smoothing length to eliminate the tensile instability that was reported in the literature $[13,14]$. The numerical simulations included the collision and vaporization of droplets. These two phenomena are critical to the mixture distribution, which in turn determines ignition and the quality of combustion. The vdW equation of state was integrated into the SPH formulation to predict the vapor-liquid equilibrium at high temperatures, and the numerical results were quantitatively compared with analytical data. Stable simulations of head-on and off-center binary collisions were achieved for high-velocity drops at $16.9<\operatorname{Re}<132.5$ and $1.62<\mathrm{We}<104$, an achievement not previously reported 
in the literature. This work lays the foundation for high-fidelity simulation of drop-drop and drop-wall collisions at high-temperature and high-pressure combustor conditions.

\section{SPH formulation of van der Waals fluid}

In the SPH methodology $[1,2,15]$, every field variable (scalar or vector) can be evaluated according to Eq. (1), which is a finite kernel approximation of the true smoothed estimate given by Eq. (2). Here $\mathrm{N}$ is the number of particles within the support domain. The value of the kernel interpolation approaches the true estimate as $\mathrm{N}$ becomes very large $[1,2] . W(r-r, h)$ is the smoothing kernel used for the interpolation summation. $r$ is the position vector of the particle, at which the variable is evaluated. $r$, is the position vector of the neighboring particle. $h$ is the smoothing length, or resolution scale, which determines the support of the kernel function. In this paper, the hyperbolic kernel function was used (Eq. (3)-(4)). Here, $\alpha_{\mathrm{d}}$ has the values of $1 /(7 h), 1 /\left(3 \pi h^{2}\right)$ and $15 /\left(62 \pi h^{3}\right)$ in one-, two- and three-dimensions, respectively.

$$
\begin{gathered}
F(r)=\sum_{b=1}^{N} \frac{m_{b}}{\rho_{b}} F\left(r_{b}\right) W\left(r-r_{b}, h\right) \\
F_{S}(r)=\int F\left(r^{\prime}\right) W\left(r-r^{\prime}, h\right) d r^{\prime} \\
W_{a b}=W_{a b}\left(r_{a}-r_{b}, h\right)=W(s)=\alpha_{d} \begin{cases}s^{3}-6 s+6, & 0 \leq s<1 \\
(2-s)^{3}, & 1 \leq s<2 \\
0, & 2 \leq s\end{cases} \\
s=\frac{\left|r_{a}-r_{b}\right|}{h}
\end{gathered}
$$

As mentioned earlier, a variable smoothing length $h$ was used in order to ensure that an adequate number of particles participate in the kernel evaluation. Monaghan et al. [1] proposed that $h$ should vary 
directly with the standard deviation of the distance between particles. Subsequently, they suggested that $h$ should be inversely proportional to the number of neighboring particles raised to $1 / d$, where $d$ is the number of dimensions [15]. Hernquist and Katz [14] used a similar version of this approach, in which an average value of the old and new smoothing lengths was used. In this paper, the value of $h$ varies according to Eq. (5) [16], which is a generalized version of that used by Monaghan and Lattanzio [17]. Here, $m_{a}$ is the individual particle mass. The ratio of $(h / i)$, where $i$ is the average inter-particle distance, remains the same or bounded within limits. The mean value is $\varsigma$ and its variance depend on the local density gradients and the concentration of particles. In order to ensure consistency and conserve momentum, a symmetrized form of the kernel function and its derivative [14] was used (Eq. (6)-(7)).

$$
\begin{gathered}
h_{a}=\varsigma\left(\frac{m_{a}}{\rho\left(r_{a}\right)}\right)^{1 / d} \\
\bar{W}_{a b}=\frac{1}{2}\left\{W_{a b}\left(r_{a}-r_{b}, h_{a}\right)+W_{a b}\left(r_{a}-r_{b}, h_{b}\right)\right\} \\
\nabla \bar{W}_{a b}=\frac{1}{2}\left\{\nabla W_{a b}\left(r_{a}-r_{b}, h_{a}\right)+\nabla W_{a b}\left(r_{a}-r_{b}, h_{b}\right)\right\}
\end{gathered}
$$

The mathematic equations governing the fluid dynamics are described in Eqns. 8-12 and are solved for obtaining the changes in momentum and internal energy of individual particles [5, 7-9, 12]. The stress tensor $S$ is formed on the basis of Eq. (10) and (11). Note that $e$ is the specific internal energy at the particle location. In addition, the vdW mechanical and calorific equations of state (Eq. (13)-(14)) were considered for calculating pressure and temperature at any location in the simulation domain.

$$
\begin{gathered}
\frac{d u}{d t}=\frac{1}{\rho} \nabla \cdot S+g \\
\frac{d e}{d t}=\frac{1}{\rho} S: \nabla u-\frac{1}{\rho} \nabla \cdot q
\end{gathered}
$$




$$
\begin{gathered}
S=-p I+\sigma \\
\sigma=\eta(\nabla u+u \nabla)+\left(\zeta-\frac{2}{d} \eta\right)(\nabla \cdot u) I \\
q=-\kappa \nabla T \\
p=\frac{\rho \bar{k} T}{1-\rho \bar{b}}-\bar{a} \rho^{2} \\
T=(e+\bar{a} \rho) / \bar{k}
\end{gathered}
$$

To calculate the density distribution of the fluid, Eq. (15) was employed. In order to maintain variational consistency, a symmetrized SPH discretization for the governing equations (Eq. (16)-(18)) is required [18].

$$
\begin{gathered}
\rho_{a}=\sum_{b} m_{b} \bar{W}_{a b} \\
\frac{d u_{a}}{d t}=\sum_{b} m_{b}\left(\frac{S_{a}}{\rho_{a}^{2}}+\frac{S_{b}}{\rho_{b}^{2}}\right) \cdot \nabla_{a} \bar{W}_{a b}+g_{a} \\
\frac{d e_{a}}{d t}=\sum_{b} m_{b}\left(\frac{S_{a}}{\rho_{a}^{2}}+\frac{S_{b}}{\rho_{b}^{2}}\right):\left(u_{a}-u_{b}\right) \nabla_{a} \bar{W}_{a b}-\sum_{b} m_{b}\left(\frac{q_{a}}{\rho_{a}^{2}}+\frac{q_{b}}{\rho_{b}^{2}}\right) \cdot \nabla_{a} \bar{W}_{a b} \\
q_{a}=-\kappa \sum_{b} 2 m_{b} \frac{T_{b}-T_{a}}{\rho_{a}+\rho_{b}} \nabla_{a} \bar{W}_{a b}
\end{gathered}
$$

The evaluation of momentum and energy changes due to the cohesive pressure term, $-a \rho^{2}$, requires a special treatment [5]. In order to remain stable during the vdW drop evolution, it is necessary to use a larger smoothing length to account for the contributions of the attractive pressure force [5, 7-9, 12]. This requires a separate discretization and calculation as described by Eq. (19) and (20), in which a 
smoothing length, twice of that used for all other calculations, was employed. All of the simulations in this paper were performed using a leap-frog time-step integration scheme.

$$
\begin{gathered}
\left(\frac{d u_{a}}{d t}\right)^{c p}=2 \bar{a} \sum_{b} m_{b} \nabla_{a} \bar{W}_{a b}^{H=2 h} \\
\left(\frac{d e_{a}}{d t}\right)^{c p}=\bar{a} \sum_{b} m_{b}\left(u_{b}-u_{a}\right) \cdot \nabla_{a} \bar{W}_{a b}^{H=2 h}
\end{gathered}
$$

\section{Numerical simulation results}

\subsection{Formation and oscillation of vdW drops}

The SPH method, developed in this study, was first tested to predict the thermodynamic and mechanical stability of the vdW drop. The evolution of a body of liquid from the initial rectangular, nonequilibrium configuration into a circular shape was predicted, as shown in Fig. 1. The vdW parameters were $\bar{a}=2.0, \bar{b}=0.5$ and $\bar{k}=1[5,7-9,10-12]$. A rectangular distribution of $2000(20 \times 100) \mathrm{SPH}$ particles with initial inter-particle distance of 0.75 units was initialized with a uniform temperature distribution, non-dimensional $T=0.2$. The simulation was run for a non-dimensional time duration of 1000. The simulation timestep was 0.005, and every particle had a unit mass. At the first stage (Fig. 1a), the surface tension pulled the liquid from both ends of the rectangle to form a dumb-bell shape. Due to inertia, the liquid continued to be compressed, and pushed out in the vertical direction (Fig 1b-1c). Then the surface tension pulled the outer lobes to the center, and further pushed some liquid particles horizontally to both ends. The liquid continued to oscillate until the momentum of oscillation died down (Fig 1d-1f), and finally a stable drop was formed. The numerical results indicate that the present model can simulate the dynamics of a liquid drop and the detailed evolution of the liquid surface when subject to the vdW force. The simulation results are reasonable based on the understanding of drop collision physics, and that the trend of collision outcomes with respect to impact velocity agree qualitatively with the literature results. Note that all values used in this paper are non-dimensional. 


\subsection{Binary collision of vdW drops}

In the literature [7, 8, 10-12], it has been argued that the inequalities given by Eq. (21) and (22) should be satisfied by the initial configuration of the SPH particles for stable evolution. Eq. (22) does not allow the kinetic pressure to be negative, nor does it prevent the acoustic velocity from approaching singularity. Eq. (21) ensures that the acoustic velocity is not imaginary. In the simulation of the evolution of a rectangular drop (shown in Fig. 1), the initial liquid configuration in conjunction with the vdW parameters and temperature resulted in a supercooled drop with no spinodal decomposition.

$$
\begin{gathered}
\bar{k} T>2 \bar{a} \rho(1-\rho \bar{b})^{2} \\
\rho<1 / \bar{b}
\end{gathered}
$$

For the purpose of simulating binary liquid drop collisions, a stable liquid drop was initially formed using the aforementioned parameters with an initial square distribution of 400 particles $(20 \times 20$ particles). A mirror image of this drop was created, and equal, but opposite, velocities were imposed on the particles of the two drops. The initial separation between the two drops was 18 units. Initially a few cases were tested to observe the collision outcomes. In the early testing, stable results were obtained for relative velocity $u_{r e l}<1.0$, but the simulation became unstable for higher relative velocity. The instability was caused by an increase in the magnitude of density near the collision interface, which exceeded that allowed by Eq. (22). This resulted in the kinetic pressure becoming negative and the acoustic velocity becoming unbounded. In other words, the von Neumann stability of the system was violated, along with its physical reality.

To mitigate this problem, a near saturated state can be stipulated, and/or a different value of the parameter $\bar{b}$ can be introduced, so that the initial drop center density is not too close to the critical value of $1 / \bar{b}$. Using this method, a stable drop was formed using $\bar{b}=0.3, T=0.6$ and initial inter-particle separation of 0.65 units. The resulting drop had a center density of 2.6 , which is $22 \%$ less than the

critical value of 3.33. Using these new parameters, drop collision simulations were performed for 
relative velocity of $0.5,2.0,3.0$ and 4.0. These numbers corresponded to $R e$ of $16.9,67.6,101.4$ and 135.2 respectively. The surface tension of the drop was calculated using the Laplace equation, Eq. (23), which yielded a value of 5.2 for the drop radius of 6.5 units. The values of $W e$ for the four cases were $1.62,6.50,14.62$ and 104 , respectively.

$$
p_{l}=p_{g}+\frac{\gamma}{R}
$$

Selected snapshots of head-on collisions are shown in Fig. 2. Two drops approached each other from the horizontal direction. In the first two simulations $\left(u_{r e l}=0.5,2.0\right)$, coalescence was stable. Despite slight fragmentation, observed in the second simulation, nearly all of the SPH particles merged into a drop. Partial transient stretching was also observed in the second simulation, but the surface tension eventually overcame the inertial force due to the impact and pulled the two lobes together. In the third simulation $\left(u_{r e l}=3.0\right)$, stretching separation took place, forming two stable drops in the end. In the fourth simulation $\left(u_{r e l}=4.0\right)$, the stretching effect was more pronounced, leading to the formation of four distinct droplets.

Off-center collisions of two identical drops were also simulated, as illustrated in Fig. 3. The impact parameter was 0.385 . The impact parameter is defined as the ratio of the vertical distance between the centers of two drops to the drop diameter. Except in simulation $u_{r e l}=0.5$, where coalescence occurred, the collisions resulted in stretching separation. Even in simulation $u_{r e l}=2.0$, which had stable coalescence for head-on collision (Fig. 2), two separate drops were created in the off-center collision (Fig. 3). Similarly, in simulations $u_{r e l}=3.0$ and 4.0, more satellite droplets were produced in comparison to head-on collision. This can be attributed to the additional effect of the net angular momentum, which stretches the colliding drops further, and also to the smaller interface area formed just after collision such that the liquid was weakly connected.

\subsection{Vapor-liquid equilibrium}


In simulating vapor-liquid equilibrium of a vdW drop, a stable drop was initialized, similar to that used in the binary collision. However, it was found that a higher resolution was needed to resolve the vapor phase. Thus, a drop was represented using 900 particles. To achieve the desirable drop temperature prior to the vaporization event, energy was added to all the SPH particles in small increments for a duration of 200 units of time. Then, the particles were allowed to vaporize to achieve vapor-liquid equilibrium in the next 200 time units. This methodology was used to prevent the liquid drop from fragmenting into many small droplets when a high initial temperature was imposed at the start of simulation. In this way, a stable round liquid drop with distinct liquid and vapor regions was achieved. The rate of energy addition was varied to achieve different temperatures. The size of the periodic boundary was adjusted to facilitate simulation stability while producing dense vapor near the critical temperature.

The analytical saturation line was first obtained by using Maxwell's equal-area rule and the vdW equation of state to iteratively solve for saturation properties (Figs. 4 and 5). As seen in Fig. 4, when a fixed smoothing length was used in the simulation, for temperatures below the critical point, the vapor density obtained from SPH simulations was found to deviate from the analytical value (Fig. 4), and this error increased at low temperatures. This was caused by the use of a fixed smoothing length, as a result of which the particle interaction was reduced. Eventually at a low enough temperature, the vapor density reached the limiting point at which there was no particle interaction. This problem was solved with the use of a variable smoothing length (Eq. (5)) with $\varsigma=2.2$ and $d=2$.

The improved vapor density prediction is evidenced in Fig. 4. However, even with this treatment, discrepancies between the predicted and analytical values still existed for reduced temperature below 0.5. To improve the accuracy further, a higher resolution using more SPH particles was needed for more adequate particle interaction. Using 3600 particles to represent a liquid drop together with variable smoothing length approach, a liquid-vapor ratio of about 140 could be predicted with sufficient accuracy (Fig. 5). The distribution of the SPH particles, and the spatial variation of density using 3600 particles 
was illustrated in Fig. 6. It can be seen that as temperature increased, more vapor was produced. At low temperatures, the liquid drop had a clear surface. As temperature approached the critical temperature, there was no clear distinction between the liquid and gas phases. The simulations, and the observations found in the literature, agree and indicate that the liquid-gas interface becomes blurred at supercritical environments [20].

Note that recovering the thermodynamic data is critical because the present method does not employ any engineering model but only utilizes the equation of state. Fig. 6 illustrates the distribution of particles at saturation conditions for different saturation temperatures, which is quantitatively depicted in Fig. 5. This work is the first step towards a larger goal in predicting high-pressure vaporization without the need of using models since the fundamental fluid and energy equations are solved directly using the present SPH method. Quantitative validations of realistic fuels can be carried out in the future.

\section{Conclusion}

The SPH method offers a convenient approach to simulating the dynamics of fluids with large deformations, without the need for a transport equation to track the interface. When coupled with a van der Waals equation of state, this method can predict the oscillation of an unstable liquid until it reaches its equilibrium in shape. The problem of tensile instability, observed previously, can be resolved by use of a hyperbolic kernel function that has only one sign for its second-order derivative. The capability of the numerical method, presented in this study, to predict binary collisions of high-velocity drops and the vapor-liquid equilibrium at high temperatures was demonstrated.

Successful numerical results for drop collisions of the Weber number up to 104 were obtained. Collisions with such high impact energy were shown to result in stretching separation, generating numerous satellite droplets. Furthermore, results from vapor-liquid equilibrium calculations showed that the present modeling approach can successfully predict the saturation properties described by a cubic equation of state. The results also emphasized the importance of using a variable smoothing length in 
order to accurately capture the physics of a sparse vapor media. The predicted distribution of liquid and vapor, as a function of temperature, is consistent with the observation in the literature. As the critical temperature was approached, the interface between the liquid and the vapor become indistinguishable.

\section{Acknowledgment}

Supports from the U.S. Army Research Laboratory are acknowledged. This work was supported in part by high performance computer time and resources from the DoD High Performance Computing Modernization Program (HPCMP) FRONTIER Award. The Army project is titled "Petascale High Fidelity Simulation of Atomization and Spray/Wall Interactions at High Temperature and Pressure Conditions."

\section{References}

[1] R. A. Gingold and J. J. Monaghan, Mon. Not. Royal Astronomical Society, 181 (1977) 375-389

[2] L. B. Lucy, The Astronomical Journal, 82 (12) (1977)

[3] J. J. Monaghan, Journal of Computational Physics, 159 (2000) 290-311

[4] J. P. Gray, J. J. Monaghan and R. P. Swift, Computer methods in applied mechanics and engineering, 190 (2001) 6641-6662

[5] S. Nugent and H. A. Posch, Physical Review E, 62 (4) (2000)

[6] J. W. Swegle, D. L. Hicks and S. W. Attaway, Journal of Computational Physics, 116 (1995) 123134

[7] Y. Melean, L. D. G. Sigalotti and A. Hasmy, Computer Physics Communications, 157 (2004) 191200

[8] L. D. G. Sigalotti, J. Klapp, E. Sira, Y. Melean and A. Hasmy, Journal of Computational Physics, 191 (2003) 622-638

[9] X. Yang, M. Liu and S. Peng, Computers \& Fluids 92 (2014) 199-208

[10] L. D. G. Sigalotti, J. Troconis, E. Sira, F. Pena-Polo and J. Klapp, Physical Review E, 90 (2014) 
[11] L. D. G. Sigalotti, J. Troconis, E. Sira, F. Pena-Polo and J. Klapp, Physical Review E, 92 (2015)

[12] Y. Melean and L. D. G. Sigalotti, International Journal of Heat and Mass Transfer, 48 (2005) 40414061

[13] M. Steinmetz and E. Muller, Astronomy and Astrophysics, 268 (1993) 391-410

[14] L. Hernquist and N. Katz, The Astrophysical Journal Supplement Series, 70 (1989) 419-446

[15] R. A. Gingold and J. J. Monaghan, Journal of Computational Physics, 46 (1982) 429-453

[16] M. Steinmetz, E. Muller, Astronomy and Astrophysics, 268 (1993) 391-410

[17] J. Monaghan and J. Lattanzio, Astronomy and Astrophysics, 149 (1985) 135-143

[18] J. Bonet and T.-S. Lok, Computational methods in applied mechanics and engineering, 180 (1999) 97-115

[19] D. S. Balsara, Journal of Computational Physics, 121 (1995) 357-372

[20] Bellan, J., Prog. Energy Combust. Sci. 26 (2000), 329-366 


\section{Figure captions}

Fig. 1. Snapshots of the evolution of a non-equilibrium rectangular drop

Fig. 2. Snapshots of binary drop head-on collisions: (a) $\mathrm{u}_{\mathrm{rel}}=0.5, \operatorname{Re}=16.9$, We $=1.62$; (b) $\mathrm{u}_{\mathrm{rel}}=2.0$, $\operatorname{Re}=67.6, \mathrm{We}=6.50 ;(\mathrm{c}) \mathrm{u}_{\mathrm{rel}}=3.0, \mathrm{Re}=101.4, \mathrm{We}=14.62 ;(\mathrm{d}) \mathrm{u}_{\mathrm{rel}}=4.0, \operatorname{Re}=135.2, \mathrm{We}=104$.

Fig. 3. Snapshots of binary drop off-center collisions (impact parameter $b=0.385$ ): (a) $u_{\text {rel }}=0.5, \operatorname{Re}=$ $16.9, \mathrm{We}=1.62 ;$ (b) $\mathrm{u}_{\mathrm{rel}}=2.0, \operatorname{Re}=67.6, \mathrm{We}=6.50 ;$ (c) $\mathrm{u}_{\mathrm{rel}}=3.0, \operatorname{Re}=101.4, \mathrm{We}=14.62 ;$ (d) $\mathrm{u}_{\mathrm{rel}}=4.0, \mathrm{Re}=135.2, \mathrm{We}=104$.

Fig. 4. Saturation temperature-density lines (using $900 \mathrm{SPH}$ particles)

Fig. 5. Saturation temperature-density lines (using 3600 SPH particles and variable smoothing length)

Fig. 6. SPH particle distribution and density field (color bar) at equilibrium for different reduced temperatures (from top left to bottom right, $\mathrm{T}=0.440,0.566,0.713$, and 0.964 ) 